\section{Nobel award to Prelog}

PROFESSOR Vladimir Prelog of the Federal Technical High School (ETH) in Zürich has been awarded half of the Nobel Prize for Chemistry, at the age of 69 , for his work on stereochemistry. It comes to him not so much for any one outstanding discovery, but for a lifetime's work in chemistry which has produced a steady succession of significant researches combining deep thought and elegant experimental work. van't Hoff is considered as the founder of 'Chemistry in space'; Prelog has been the High Priest of this cult for the past 20 years.

Prelog was born in 1906 in Sarajevo in the Austro-Hungarian Empire. $\mathrm{He}$ studied in Prague, worked in industry, and then joined the staff of the University of Zagreb in Yugoslavia. Fortunately for Prelog and for chemistry, he found his way to Switzerland in 1941 and joined the great ETH School of Organic Chemistry. His career in Zürich was naturally influenced by his predecessor, Professor L. Ruzicka (Nobel Laureate, 1939), whom he succeeded in 1957. Prelog's structural and synthetic studies on natural products have covered a wide range, including terpenes, steroids, alkaloids and antibiotics.

A major stereochemical interest started from the preparative work of the Zürich school on medium and large rings, and developed into Prelog's studies on transannular reactions. In the spirit of his time, Prelog not only made new compounds by ingenious methods, but studied their physical properties and their reactivity, using all of the steadily increasing range of tools provided by physical chemistry.

His work in stereochemistry has included a considerable share in the study of conformations, for example, his pioneer work on asymmetric synthesis and the medium-ring work noted here. He delivered one of the earliest Centenary Lectures to the Chemical Society in London in 1949; this significantly influenced the thinking of other pioneers of conformational studies.

Prelog's ability to get to the heart of things is nowhere better illustrated than in the work on the Sequence Rule. This work, the result of a very fruitful partnership between Prelog, the late Sir Christopher Ingold and $\mathrm{Dr}$ R. S. Cahn, soon developed into something much more than a method for designating stereoisomers (the " $R$ and $S$ " system). It led Prelog to consider in a simple, but fundamental way, the



geometrical basis of stereochemistry.

The Sequence Rule papers first showed clearly and in a systematic framework of thought the nature of axes and planes of chirality. (The term "chirality", borrowed from Kelvin, has been universally accepted as a convenient term for the property of "handedness".) The need to test the Sequence Rule exhaustively during its development caused Prelog to visualise, and then to make with his students, organic compounds of widely differing types displaying stereoisomerism of kinds apparently unknown in nature, and hitherto unsuspected by organic chemists-for example, cycloenantiomers and cyclodiastereoisomers.

In all of this, Prelog has carried to its logical conclusion the concept of the tetrahedral atom (van't Hoff and Le Bel). The marriage of chemistry and geometry thus arranged by Prelog, and called by him "chemical topology" is already a happy and most fruitful one. All types of stereoisomerism can be derived from the consideration of simplexes in two- and three-dimensional space, alone or in combination.

The general principles were outlined in lectures in Amsterdam, in Dublin, in Hamburg, and in Zürich (Karrer Lecture), and were presented in a more extended from in lectures at Cornell. Some of these ideas have now appeared in a very general paper modestly entitled "Pseudoasymmetry in organic chemistry", which was followed by two detailed papers on compounds showing axes and planes of pseudoassymetry, giving some experimental 'meat' to cover the elegant geometrical skeleton. The international group characteristically addressed by Prelog in letters as Stereofreunde await with great interest and some impatience the fuller story.

Much of Prelog's recent work has been on the borders of chemistry and biochemistry, including his studies on enzymatic reactions. In addition to their supreme biochemical importance, enzymatic reactions provide a suitable terrain for thinking in terms of twodimensional chirality.

Like many continental professors, Prelog has long had close connections with industry, and he has been for some years a Director of Ciba-Geigy in Basel.

$\mathrm{He}$ is a frequent and welcome visitor to laboratories all over the world-and nowhere more welcome than in the UK. Few chemists offer more stimulating discussion to collegues of all ages and interests. $\mathrm{He}$ is always one of the liveliest disputants at any meeting, and he was one of the founding fathers of the annual Bürgenstock Conferences on Stereochemistry.

The Stereofreunde rejoice in this award to Prelog. Perhaps the pilgrimage to Stockholm will usher in an age of many-dimensional stereochemical thinking.

W. Klyne 